\title{
CONTENTS
}

\section{Plenary Lectures}

Biomechanics of aquatic micro-organisms . T. J. Pedley (1)

An investigation of scalar dispersion in grid turbulence $\ldots \ldots \ldots \ldots \ldots \ldots \ldots \ldots \ldots+$ P. E. Dimotakis, D. B. Lang, S. Lombeyda, J. Lindheirn (7)

Flow control and hydrodynamic instability

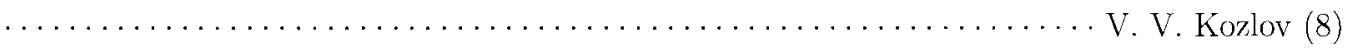

Recent progress on understanding and modelling ocean wave breaking

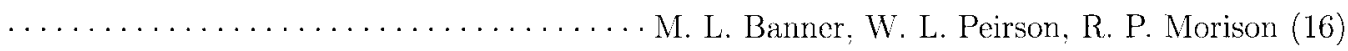

Recent progress on understanding and modeling sediment transport in coastal environment

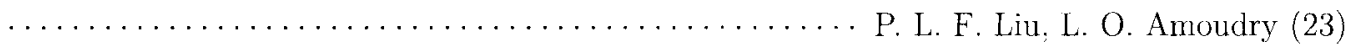

Turbulent drag reduction with surfactant additives - basic research and application to an air conditioning system ................... Kawaguchi, F. C. Li, B. Y11, J. J. Woi (29) Research progress on high-enthalpy and hypersonic flows

Numerical and experimental research on cavitating flows

C. J. Lu, Y. S. He, X. Chen, Y. Chen (45)

\section{Flow Transition and Instability}

Modeling flow transition in hypersonic boundary layer

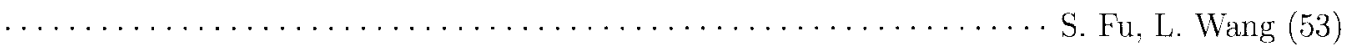

Three important theorems for flow stability

Voltage-induced flow instability and turbulence in Banana-shaped liquid crystal

$\ldots \ldots \cdots \cdots \cdots \cdots \cdots \cdots \cdots \cdots \cdots \cdots \cdots$ Y. M. Huang, L. L. Chen, F. F. Zhou, B. G. Zhai (61)

Stability analysis of boundary-layer transition using accurate velocity profiles obtained by

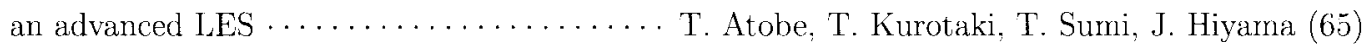

Convection in a fluid layer heated from below and subjected to time periodic horizontal accelerations

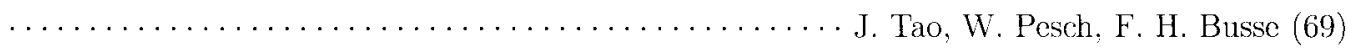

Numerical studies of flow past two side-by-side circular cylinders

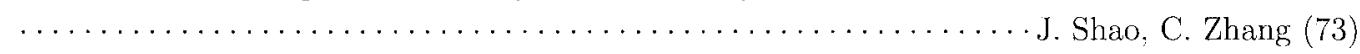

Essence of inviscid shear instability: a point view of vortex dynamics

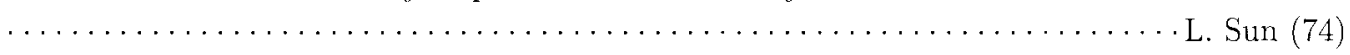

Threc-dimensional cvolution of the flow through a curved square duct

T. Watanabe, S. Yanase (75)

Numerical cstimation of the critical Reynolds number for flow past one square cylinder with symmetric geometry boundary condition .......... X. H. Wang, W. F Zh11, Z. Y. He (76)

Turbulent transition in plane Couette flows 
Solution to stability analysis in stratified liquid film flowing down an inclined heated plate

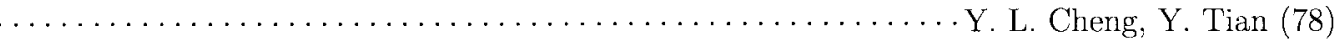

\section{Turbulence}

Overview of detached-eddy simulation for external and internal turbulent flow applications

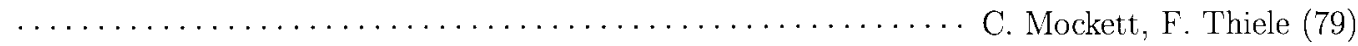

Computation of turbulence-generated noise by large-eddy simulation H. D. Yao, G. W. He, X. Zhang (83)

The vortex breakdown of a columnar vortex with axial flow

Statistical characteristics of elastic turbulence in a free-surface swirling flow $\ldots \ldots \ldots \ldots \ldots \ldots \ldots \ldots \ldots$ F. C. Li, M. Oishi, Y. Kawaguchi, N. Oshima, M. Oshima (91)

Large eddy simulation of a self-preserving turbulent jet using high-order schemes

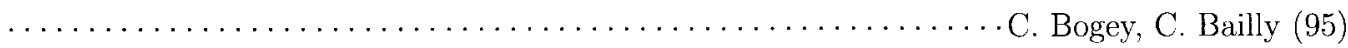

Large-eddy simulation of turbulent combustion using SOM and EBU SGS combustion models

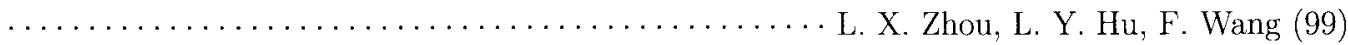

Large-eddy simulation of a turbulent buoyant flame interacting with droplets

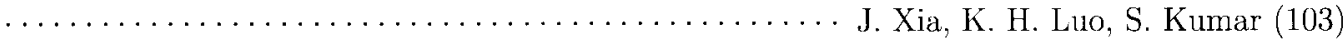

Large eddy simulation of heavy gas dispersion around an obstacle

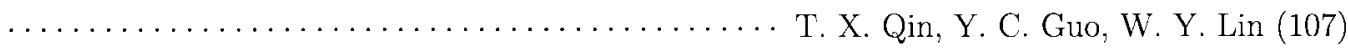

Multiscale large eddy simulation of scalar transport in turbulent channel flow

C. X. Xu, Z. Y. Wang, G. X. Cui, Z. S. Zhang (111)

Large eddy simulation of urban heat island phenomenon

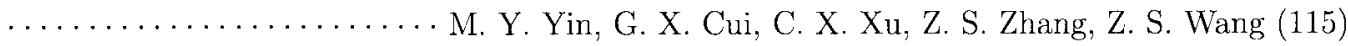

LES modeling of a swirling diffusion flame and validation of different SGS stress and combustion

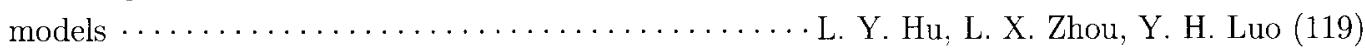

Experimental and large-eddy simulation studies on three-dimensional vortices produced by an impinging transverse jet $\cdots \cdots \cdots \cdots \cdots \cdots \cdots \cdots$ J. Y. Fan, Y. Zhang, D. Z. Wang (123)

Prediction of particle distribution in isotropic turbulence by large-eddy simulation

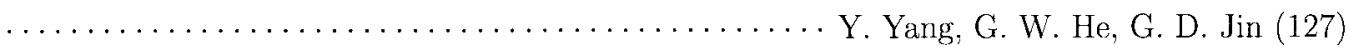

Large eddy simulation of Re's influence on the quasi-pcriodic motions of the turbulent flow over a

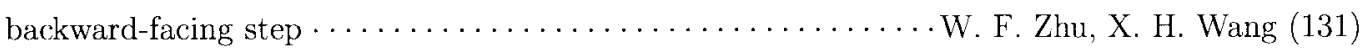

Numerical simulation of convective heat and mass transfer in a two-layer system

$\ldots \ldots \ldots \cdots \cdots \cdots \cdots$ B. I. Myznikova, V. A. Kazaryan, E. L. Tarunin, I. I. Wertgeim (134)

Modeling and simulation of coaxial jet flow

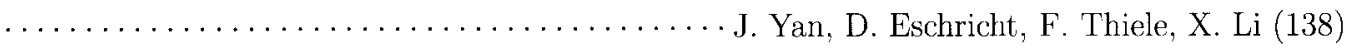

Particle settling behavior in turbulent flow generated by oscillating grid

Q. Zhou, N. S. Cheng (142)

Analysis on turbulent flow in the impeller of chemical pump

M. G. Yang, D. Liu, H. F. Gu, C. Kang, H. Li (146)

Mixing enhancement of a 2D supersonic mixing layer induced by inflow periodic temperature

excitation $\cdots \cdots \cdots \cdots \cdots \cdots \cdots \cdots \cdots \cdots \cdots \cdots \cdots$ M. B. Sun, Z. G. Wang, J. H. Liang (150)

Computational prediction of local distorted flow in turbocharger

............ J. Yao, Y. F. Yao, P. J. Mason, T. Zhang, F. J. G. Heyes, P. E. Roach (154) 
Analytical calculations of Eulerian and Lagrangian time correlations in turbulent shear flows

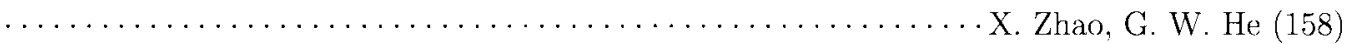

Near-field mixing characteristics of turbulent jet issuing from a notched-rectangular orifice plate

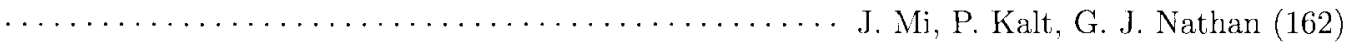

Vertical 2D algebraic-stress turbulence model of shallow water flow in $\sigma$-coordinates

$\ldots \ldots \ldots \ldots \ldots \ldots \ldots \ldots \ldots \ldots \ldots \ldots \ldots$ S. M. Wong, T. S. Li, X. G. Wu, Y. M. Shen (166)

Large eddy simulation of flow around a square cylinder

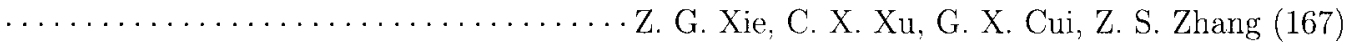

Large eddy simulation of the wind field and pollution dispersion in building array

$\ldots \ldots \ldots \ldots \ldots \ldots \ldots \ldots \ldots$ R. F. Shi, G. X. Cui, C. X. Xu, Z. S. Zhang, Z. S. Wang (168)

Experimental investigation on drag reduction of vessel model by microbubbles

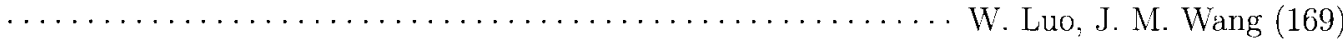

Rcynolds number effects on the flow around square cylinder based on lattice Boltzmann method

$\ldots \ldots \ldots \ldots \ldots \ldots \ldots \ldots \ldots \ldots$ T. C. Liu, Y. J. Ge, F. C. Cao, Z. Y. Zhon, W. Zhang (170)

DNS and LES of turbulent channel flow with hydrophobic surface

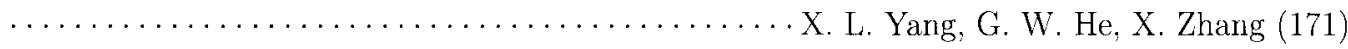

Direct numerical simulations of turbulent channel flows with moving wall

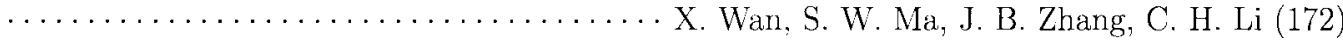

Experimental investigations of forerunners at the fronts of localized disturbances of a

straight wing boundary layer ......... V. N. Gorev, M. M. Katasonov, V. V. Kozlov (173)

The relaxation oscillation of turbulent convection in rotating cylindrical annulus

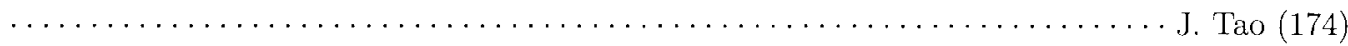

Study on the flow structures and pollutant dispersion of a moving car

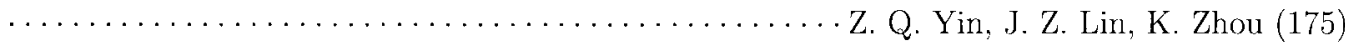

Correction on drag coefficient in simulation of fast fluidized beds

L. M. Zou, Y. C. Guo (176)

Numerical simulation of the scalar dissipation rate in the non-premixed turbulent combustion $\cdots \cdots \cdots \cdots \cdots \cdots \cdots \cdots \cdots \cdots+$ Y. F. Liu, Y. T. Zhang, H. S. Tian, J. J. Qin (177)

\section{Aerodynamics and Gas Dynamics}

The study of Reynolds number effects on the behaviors of axisymmetric vortices flow

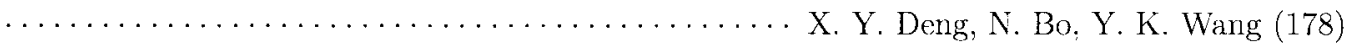

Mixing intensification by electrical discharge in high-speed flow

$\ldots \ldots \ldots \ldots \ldots \ldots \ldots \ldots \ldots \ldots \ldots \ldots \ldots \ldots \ldots$. Y. I. Isaenkov, S. B. Leonov, M. N. Shneider (182)

Measurement on asymmetric transition of a hypersonic boundary layer using a high frequency thin-film sensor system $\cdots \cdots \cdots \cdots \cdots \cdots \cdots$ Z. X. Bi, Q. Shen, Z. F. Zhang, C. H. Wu (186)

Supersonic rupture's shock control by electrical discharge ................. S. B. Leonov, V. N. Sermanov, V. R. Soloviev, D. A. Yarantsev (190)

Formula for upstream pressure, nozzle geometry and frequency correlation in shedding/discharging cavitation clouds determined by visualization of submerged cavitating Jet

E. A. F. Hutli, M. S. Nedeljkovic (194)

The Numerical Research on the the transition of the Three-Dimensional Supersonic spatial developing Mixing Layer when $M c=0.5 \cdots \cdots \cdots \cdots \cdots \cdots \cdots$ Q. Li, X. B. Deng, H. X. Zhang (198)

The study of determinacy of asymmetric vortices over slender body at post-critical Reynolds numbers.$\cdots \ldots \ldots \ldots \ldots \ldots \ldots \ldots \ldots \ldots \ldots \ldots$. W. Tian, X. Y. Deng, B. Wu, Y. K. Wang (202) 
Receptivity to frec-stream disturbance waves for blunt conc axial symmetry hypersonic boundary layer

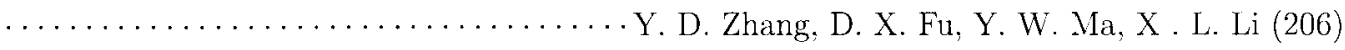

Theoretical and numerical study of vortex-wake flow phenomenon generated from stack of elliptical

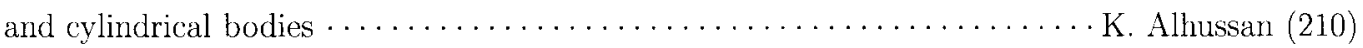
Some remarks on CFD drag prediction of an aircraft model

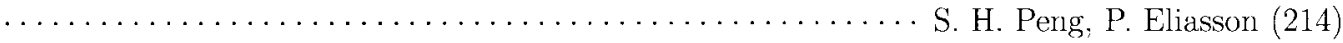

Numerical simulation of transonic buffet and flow bifurcation over airfoils

A. Kuz'min (218)

Decaying of flow separation from a low Reynolds number airfoil with high frequency perturbations H. J. Zhang, X. Y. Shi, Y. Zhou, L. Cheng, Z. D. Su (222)

Experimental investigation on the longitudinal aerodynamic performance of common swift's wing

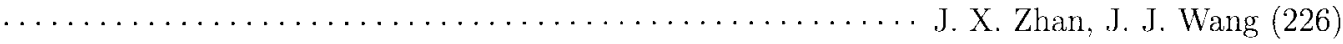

Inception of asymmetric vortex flow over slender body

B. C. Cao, B. Wang, X. Y. Deng (230)

Oil flow visualization of Reynolds number effect on asymmetric vortices at forebody

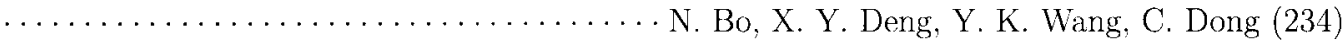

Method research for flow and mixing process of HYLTE nozzle of DF chemical laser

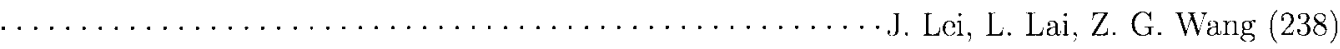

Effect of hydrodynamic development on flow and heat transfer characteristics in an

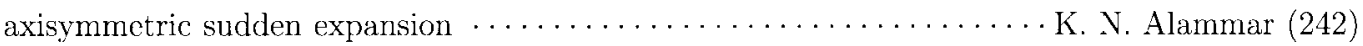

Threc-dimensional simulation of detonation waves H. S. Dou, B. C. Khoo, H. M. Tsai (245)

Study on icing test scaling law

Hybrid RANS/LES simulation of scalar transport of slot injection into a supersonic stream

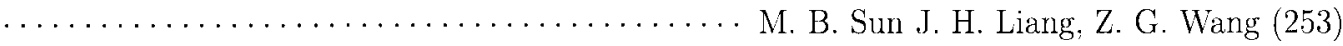

Effect of wire trips on the flow over slender body at high angle of attack

$\ldots \ldots \ldots \ldots \ldots \ldots \ldots \ldots \ldots \ldots \ldots \ldots \ldots \ldots \ldots \ldots \ldots$. Y. Chen, X. Y. Deng, Y. K. Wang (257)

A numerical study on internal and external flow fields of synthetic jet actuator

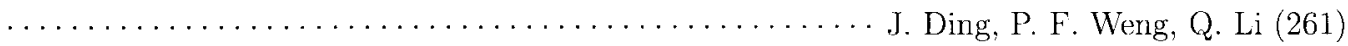

Measurements of the optical phase distortion across a supersonic flow field

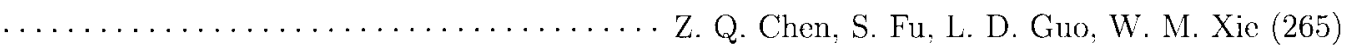
A new efficient high-resolution method for non-linear problems in fluid mechanics

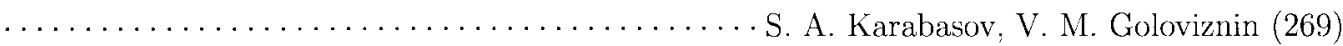

Direct numerical simulation on stability of a supersonic mixing layer flow

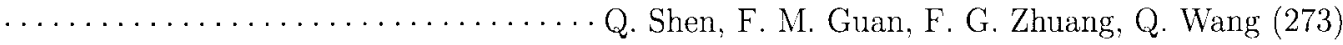
Effect of ablation on heat transfer \& performance of an axisymmetric supersonic nozzle

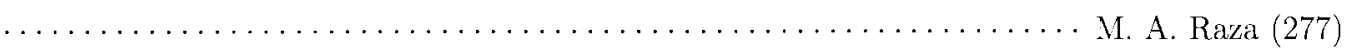
Computation of flow over a rotating body on unstructured chimera mesh

Lattice Boltzmann simulation of the flow over wavy surface

$$
\text { X. Zhang, G. W. He, S. Z. Ni (278) }
$$

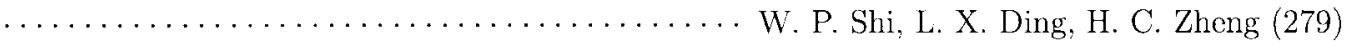

Propagation and reflection of gas waves in a close tube 
A novel design: porous boundary condition for drag reduction of three dimensional rotating body of

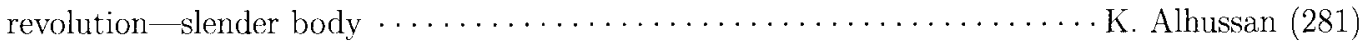
Laminar gas jets in high-temperature atmospheres

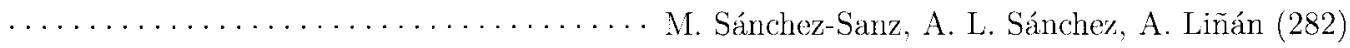

Numerical research on unsteady aerodynamics of a WIG airfoil flying over wavy water surface

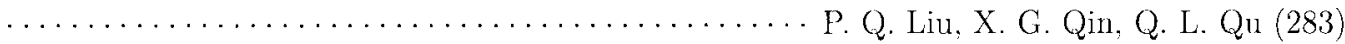

Conceptual design and numerical simulations of hypersonic waverider vehicle

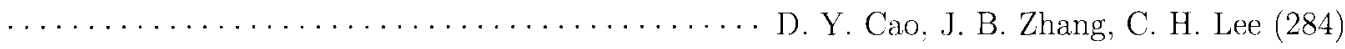

Computational study of aerodynamics of low aspect ratio wings

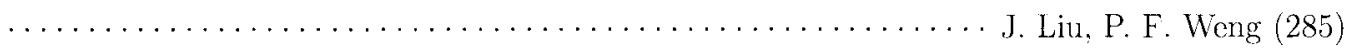

Effect of end plates on the surface pressure distribution of a given cambered airfoil: experimental study $\cdots \ldots \ldots \ldots \ldots \ldots \ldots$. K. S. V. Reddy, D. M. Shama, K. Poddar (286) Research on nozzle performance in scramjet J. P. Li, W. Y. Song, Y. Xing (287)

Numerical simulation of acrodynamic heating reduction due to opposing jet in hypersonic flow

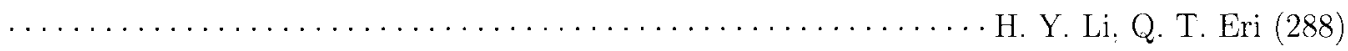

Issues on grid convergence T. Wang, Q. S. Guo (289)

Investigation of the flow in a diffusive S-duct inlet with and without secondary flow control $\ldots \ldots \ldots \ldots \ldots \ldots \ldots \ldots \ldots \ldots$ L. F. Zhang, Z. X. Liu, G. M. Guo, X. F. Wang (290)

Numerical simulation and analysis of flow-field in rotating rectangular passage

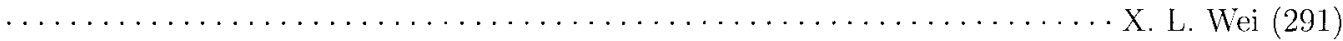

\section{Hydrodynamics}

Fully-nonlinear computation of water surface impact of axisymmetric bodies

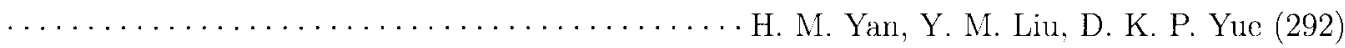
Analysis of wave passing a submerged breakwater by a scaled boundary finite element method

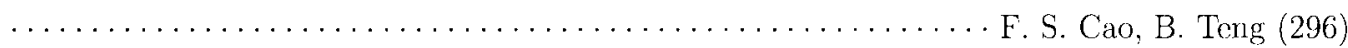

Relationship between pressure fluctuations on the bed wall and free surface fluctuations in weak hydraulic jump …K. Onitsuka, J. Akiyama, M. Shige-eda, H. Ozeki, S. Gotoh, T. Shiraishi (300) Wave loading on floating platforms by internal solitary waves

Laboratory measurements and numerical simulations of internal solitary waves in a shear flow

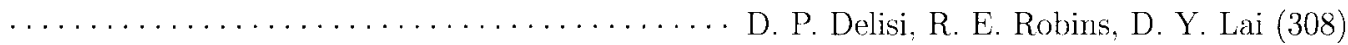

Stcady ship waves due to a simple source in a viscous fluid

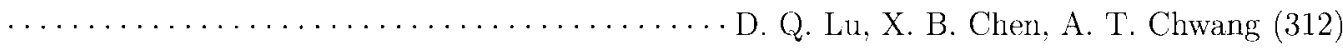

The effect of curvature on the slamming force in water impact of a WIG craft

Experimental studies of groupiness variations of waves propagating over a submerged curvilinear sill

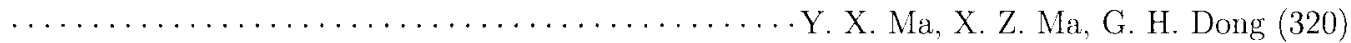

Numcrical analysis of incompressible viscous flows interacting with flexible structures D. C. Wan (324)

Development of a 3D free surface capturing code for coastal engineering flow problems

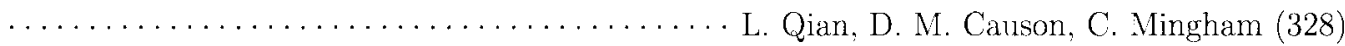


Experimental study on response of submarine pipeline over flat beds in steady flow $\ldots \ldots \ldots \ldots \ldots \ldots \cdots \cdots \cdots$ Y. Sha, Y. X. Wang, G. Y. Wang, Z. W. Li, Z. J. Chen (332) Simulation of surface pressure induced by vortex/body interaction $\ldots \ldots \ldots \ldots \ldots \ldots \ldots$ M. He, M. Islam, B. Veitch, N. Bose, M. B. Colbourne, P. Liu (336) Investigation of supercavitating multiphase flow structures

X. B. Li, G. Y. Wang, M. D. Zhang (340) Analysis of monitoring data for the safety control of dams using neural networks

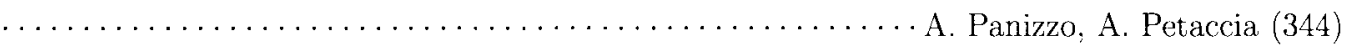

Experimental research on character of ventilated supercavity

....................... Y. J. Wei, W. Cao, C. Wang, J. Z. Zhang, Z. Z. Zou (348)

Validation of HEM based cavitation for cavitation flows around disk

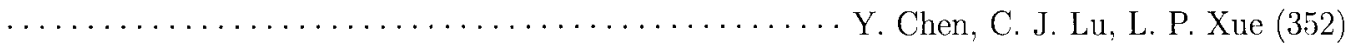

An efficient boundary fitted non-hydrostaticmodel for free-surface flows

A. Ahmadi, P. Badiei, M. M. Namin (356)

Nonlinear analysis of ship's large amplitude roll with zero speed in beam waves

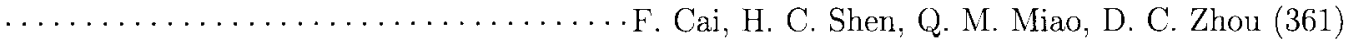

Two-dimension numerical internal wave tank for Navier-Stokes equation model in the stratified fluid G. Wei, Y. X. You, X. B. Su (364)

Characteristics of flow fields induced by interfacial waves in two-layer fluid

Y. T. Yuan (368)

Numerical simulation of circular disk entering water by an axisymmetrical SPH model in cylindrical

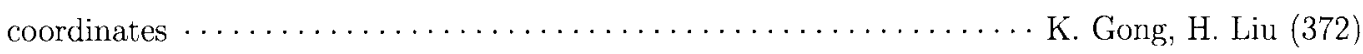

Numerical simulation of air bubble characteristics in stationary water

C. X. Zhang, Y. X. Wang (376)

Action of ship waves on a verticial cylinder in front of a vertical wall

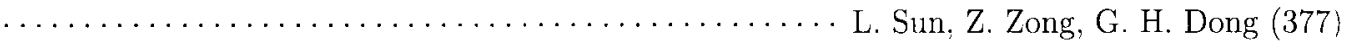

Nonlinear analysis of airship envelop acrolasticity

Experimental study of the surface wave around hydrofoils J. M. Liu, C. J. Lu, L. P. Xue (378)

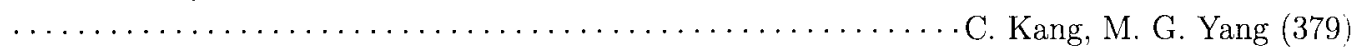

The three dimensional numerical simulation of vertical jet in waves

Numerical study of flow characteristics with free surface using turbulence model L. R. Yuan (380)

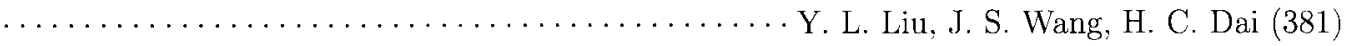

Effect of river training project on hydrodynamics flow circumstances by $2 \mathrm{D}$ finite element numerical model …... B. Zou, D. F. Li, H. J. Hu, H. W. Zhang, L. H. Lou, M. Chen, Z. Y. Lv (382) Natural boundary element method for Stokes problem of exterior circular domain $\cdots \cdots \cdots \cdots \cdots \cdots \cdots \cdots \cdots \cdots \cdots \cdots \cdots \cdots$ W. H. Peng, Z. Z. Dong, G. H. Cao, H. M. Zhao (383)

Large eddy simulation of flows near a groin in a straight open channel

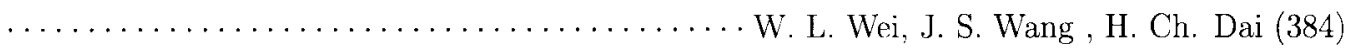

A new constitutive equation of Reynolds stress

Q. Y. Chen, H. G. Kang (385)

Shape design and experiment research of the supercavitating underwater high-speed projectile W. J. Ying, J. Hou, P. Wei (386) 


\section{Geophysical and Environmental Fluid Mechanics}

Vortex-wave interaction on a sphere

R. B. Nelson, N. R. McDonald (387)

Numerical simulation of partial-penetrating flow in horizontal convection

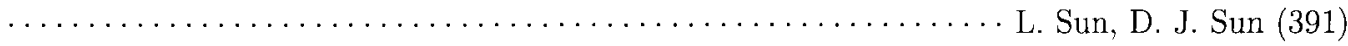

Statistical mechanics of quasi-geostrophic mono- and poly-disperse point vortex systems

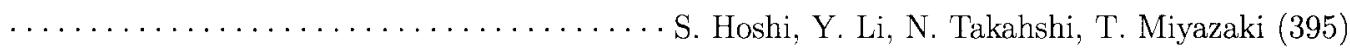

Numerical investigation of fire plume in a homogeneous shear flow

Y. Y. Liu, S. Fu (399)

Spatial variability of the surface wave field along the Washington state coast derived from synthetic

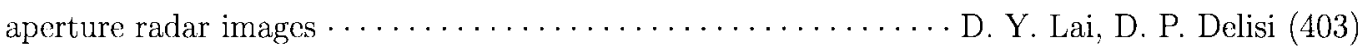

Computational modeling of aerosol hazard arising from the opening of an anthrax letter in an

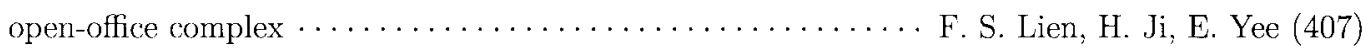

Numerical simulations of Tsunami generation by using Boussinesq equations

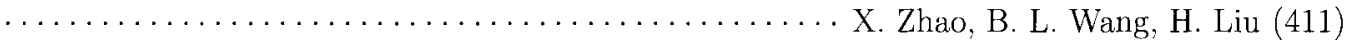

Baroclinic structure of oceanic rings

L. Zhang, C. Sun (412)

Solution of two-dimensional free surface problems based on finite element program generator B. X. Wu, S. Wan, N. N. Chen (413)

Application of the three-dimensional environmental fluid dynamics code model in Manwan reservoir

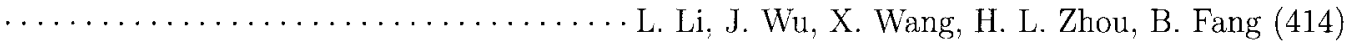

The role of flood surge plays in shaping tidal flat

X. Q. Du (415)

\section{Industrial Fluid Mechanics}

Numerical simulation of laminar circular and noncircular jets in cross-flow

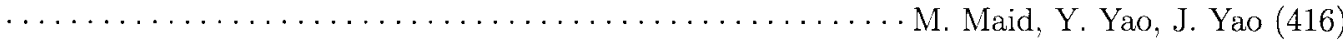

Numerical simulation of three-dimensional flow ficld in quadrate stirred tanks

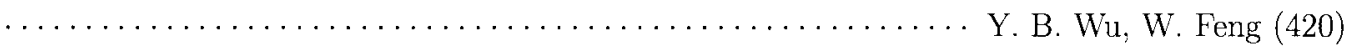

Tubular-type hydroturbine performance for variable guide vane opening by CFD … Y. T. Kim, S. H. Nam, Y. J. Cho, Y. C. Hwang, Y. D. Choi, C. D. Nam, Y. H. Lee (424)

CFD analysis for the performance of cross-flow hydraulic turbine with the variation of blade angle $\ldots \ldots \ldots \ldots \ldots \ldots \ldots \ldots$ Y. D. Choi, J. I. Lim, C. G. Kim, Y. T. Kim, Y. H. Lee (428)

An algorithm for coarse particle sedimentation simulation by Stokesian dynamics

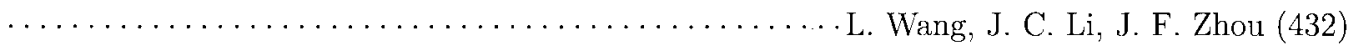

Numerical simulation of steady and filling process of low temperature liquid propellants pipeline

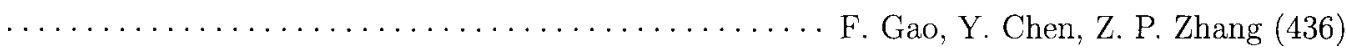

Effects of geometrical parameters and physical properties variation on transient natural convection and conduction of high Prandtl number fluid in enclosures

O. Younis, J. Pallares, F. X. Grau (440)

Nonlinear aerodynamic forces on bridge decks due to transverse sinusoidal fluctuation of wind

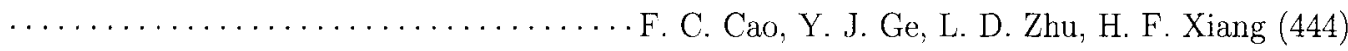

Numerical investigation of the circumferential grooved casing treatment as well as analyzing the mechanism of improve stall margin ............ H. G. Zhang, W. L. Chu, Y. H. Wu (448) 
TBCC engine inlet design and ramp angle optimization

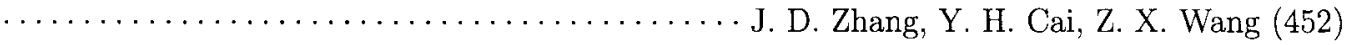

Experimental study of a fractal flowmeter in a pipe

C. H. A. Chong, S. B. M. Beck, F. C. G. A. Nicolleau (456)

Effects of rainfall infiltration on the stability of soil slopes ․ J. P. Sun, Q. Q. Liu, J. C. Li (459)

Flow characteristics of butterfly valve by PIV and CFD

$\ldots \ldots \ldots \ldots \ldots \ldots \ldots \ldots \ldots \ldots \ldots \ldots \ldots \ldots$ S. W. Kim, J. H. Kim, Y. D. Choi, Y. H. Lee (463)

Experimental investigation on momentumless wake and its application in reduction of unsteady statorrotor interaction $\cdots \cdots \cdots \cdots \cdots \cdots \cdots \cdots \cdots \cdots \cdots \cdots \cdots$, Y. D. Wu, X. C. Zhu, Z. H. Du (467)

Dynamic voids measurement during geysering phenomenon in a natural circulation loop

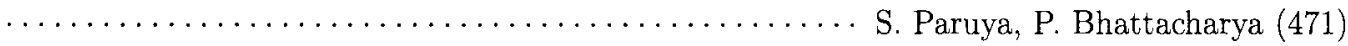

Experimental investigation on the property of high-speed ventilated supercavitation

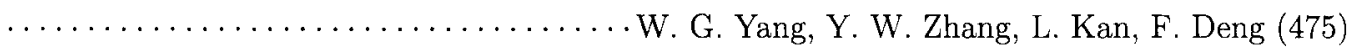

Bionic concept applied to flow slab design of PEMFC

C. T. Wang, P. C. Chang (479)

The mechanism of stall margin improvement in a centrifugal compressor with the air bleeding circumferential grooves casing treatment $\ldots \ldots \ldots \ldots \ldots$. P. Gao, W. L. Chu, Y. H. Wu (482)

Un-pairing and pairing mechanisms of cylinder's wakes at low Reynolds number

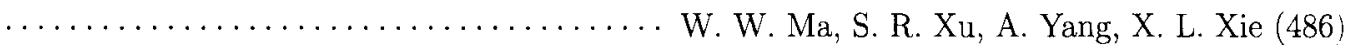

Thermal efficiency of well bore during steaming with bare tubing without packer

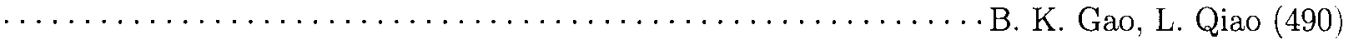

Research on optimizing design for diffuser-tower structure of primary fan in shaft

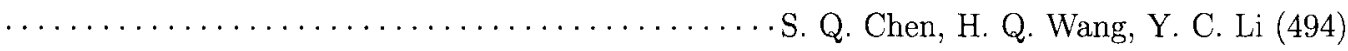

Application of hydrodynamics in design of flotation deinking cell

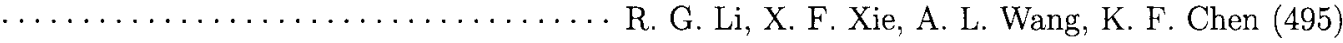

Effect of mixing chamber structure on a steam ejector performance

Force control of a square cylinder in cross flow Z. D. Su, Y. Liu, H. J. Zhang, D. F. Zhang (497) Study on the thermodynamic characteristics of the high-pressure gas in reciprocating mechanism

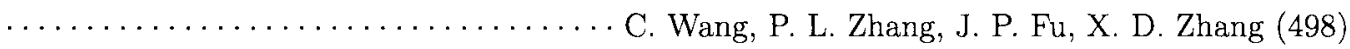

Study on supercharging effect in artillery recoil mechanism and the effects

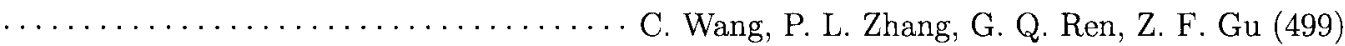

Characterization by proper-orthogonal-decomposition of flow field around bridge slotted decks

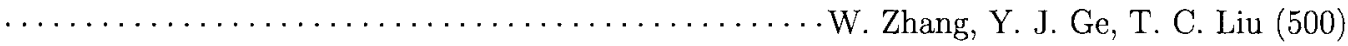

Local loss coefficient of laminar flow through sudden enlargement in circular pipes

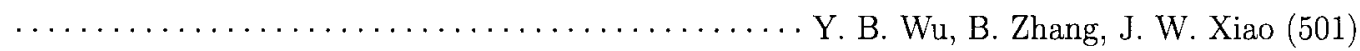

Numerical prediction of flow and heat transfer on lubricant supplying and scavenging flow path of an aero-engine lubrication system … S. Q. Huang, Y. G. Lv, L. F. Zhang, Z. X. Liu, T. Xu (502)

Experimental investigation and analysis of an axial compressor stage with $45^{\circ}$ circumferential inlet

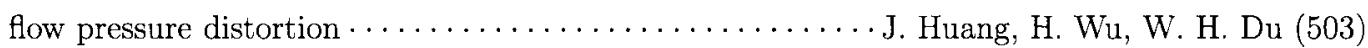

Off-design performance analysis of multi-stage transonic axial compressors 
An investigation on the effect of the hot end plugs on the efficiency of the Ranque-Hilsch vortex tube

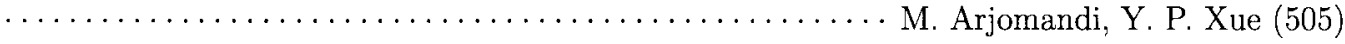

\section{Multiphase Flows, Non-Newtonian Flows and Flows in Porous Media}

Analysis of fractional element of viscoelastic fluids using Heaviside operational calculus K. Q. Zhu, K. X. Hu, D. Yang (506)

Discussion of the problems of nonlinear water wave diffraction around porous vertical circular cylinder

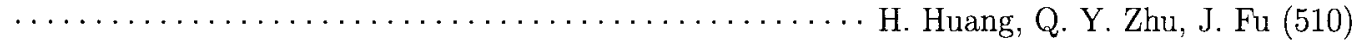

Numerical modelling of wave interaction with porous structures

$\cdots \cdots \cdots \cdots \cdots \cdots \cdots \cdots \cdots \cdots \cdots \cdots \cdot$. Gao, D. M. Ingram, D. M. Causon, C. G. Mingham (514)

Fluid flow simulation in random porous media at pore level using lattice Boltzmann method

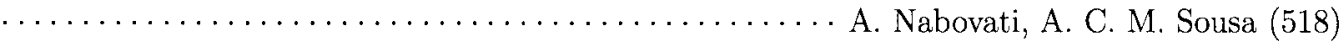

Absorption of microdrops: effect of multi-layer porous media structure parameters .................. Y. D. Varlamov, Y. P. Meshcheryakov, M. R. Predtechensky (522)

Research on flow shift law of porous media in goaf base on the unsteady airflow theory

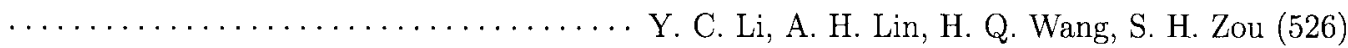

Comparisons of static, quasi-static and dynamic 3D porous media scale network models for two-phase immiscible flow in porous media $\cdots \cdots \cdots \cdots \cdots \cdots \cdots$ X. H. Zhang, Q. J. Liu, X. B. Lu (530)

Experiment and mathematical model of gas flow in low permeability porous media …........... G. Y. Zhu, L. Liu, Z. M. Yang, X. G. Liu, Y. G. Guo, Y. T. Cui (534) Resolving chemically reacting flow using moving mesh method

Droplet interactions in an imposed thermal gradient

Study on mixing field of salt tolerant polymer solution

$\ldots \ldots \ldots \ldots \ldots \ldots \ldots \ldots$ L. H. Zhang, M. G. Zhang, X. G. Li, D. Zhang, B. Jiang (546)

Lateral motion and departure of vapor bubbles in nucleate pool boiling on thin wires in microgravity $\ldots \ldots \ldots \ldots \ldots \ldots \ldots$ J. F. Zhao, S. X. Wan, G. Liu, Z. D. Li, Y. H. Lu, N. Yan (550)

Modeling of dynamic extrusion swelling using cross model

S. X. Huang, X. Chen, C. J. Lu (554)

The numerical simulation on cooling effect of microcapsulated phase change material suspension in laminar thermal developing section $\cdots \cdots \cdots \cdots \cdots \cdots \cdots$ P. Q. Liu, J. Jin, G. P. Lin (558)

PIV experiment of the gas-liquid two-phase flow within the membrane micropore aeration bioreactor

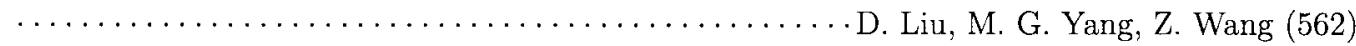

A pressure based multi-fluid algorithm for multiphase flow

$\ldots \ldots \ldots \ldots \ldots \cdots \cdots \cdots \cdots \cdots \cdots \cdots$ P. J. Ming, W. P. Zhang, G. D. Lei, M. G. Zhu (566)

Analytical solutions of $\mathrm{g}$-jitter induced double-diffusive convection with boundary collocation method Z. H. He, H. Z. Cao, Z. Y. He (570)

Numerical study on the optimization of flow and temputre field in the desulphurization spray tower $\cdots \cdots \cdots \cdots \cdots \cdots \cdots \cdots \cdots \cdots \cdots \cdots \cdots \cdots \cdot$. Zeng, L. Q. Yin, Q. Chen, Y. Wang (574)

Numerical simulations for Stochastic convection-diffusion processes in concentration fields

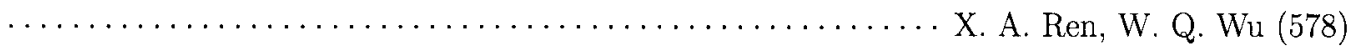

A numerical study on the characteristics of gaseous pollutant absorbed by a moving liquid aerosol

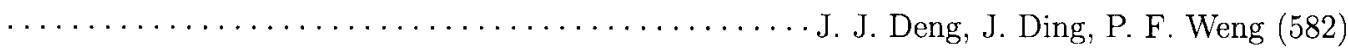


Simulation of two phase viscous flows in a solid rocket motor

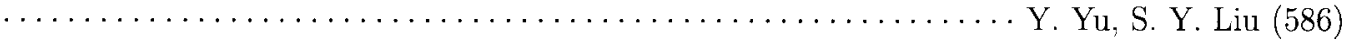

The boundary layer flow of a rivlin-ericksen fluid

K. Sadeghy, N. Khabazi, S. M. Taghavi (590)

Numerical simulation and optimization of gas-solid turbulence flow in a precalciner

Application of fluid-solid coupling theory in casing damage forecast

S. X. Mei, J. L. Xie (591)

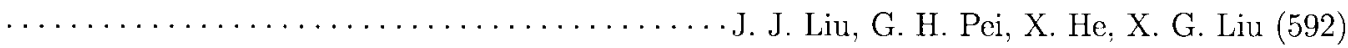

Research and test on the principle of oil temperature rise of pumps with single and double valve plate

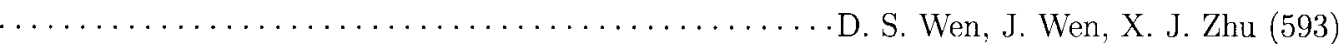

Simulation of gravity feed oil for areoplane fuel transfer system

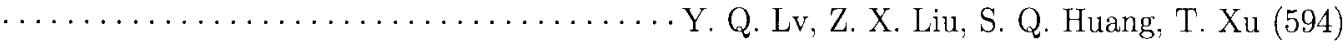

Simulation on flow behaviors of viscoelastic polymer solution in a channel with sudden contraction $\ldots \ldots \ldots \ldots \ldots \ldots \ldots$ X. G. Sui, H. J. Yin, H. Y. Zhong, L. Wang, J. Song, Z. P. Li (595)

Analysis for transient deliverability of horizontal wells $\ldots \ldots \ldots \ldots \ldots \ldots \ldots \ldots \ldots \ldots$. M. M. Pang, M. F. Li, Z. H. Luo, G. L. Zhang (596)

Boundary element method for calculating the pressure of heterogeneous reservoir $\ldots \ldots \ldots \ldots \ldots \ldots \ldots$ Y. A. Zhang, J. F. Jia, H. W. Wang, B. S. Li, Y. R. Xu (597)

3-D numerical simulation for gas-liquid two-phase flow in aeration tank

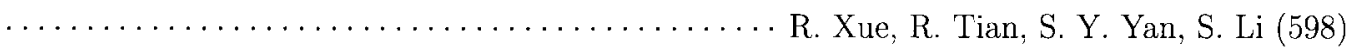

Simulation of indoor fine suspension particle deposition

K. Q. Li, G. C. Gong, S. H. Zou (599)

Axial wind-borne electrostatic spray research

… W. W. W. Wang, J. L. Wen, X. Y. Wang, T. Q. Luo, X. N. Song (600)

Permeability coefficient inversion method based on hybrid algorithm

Modeling the interface instability and mixing flow during the process of liquid explosion dissemination … L. Li, S. L. Xu, Y. J. Ren, G. R. Liu, X. B. Ren, W. J. Xie, Y. C. Li, Z. L. Wang (602)

\section{Bio-fluid Mechanics}

Impact of wall shear stress and pressure variation on the stability of atherosclerotic plaque

$\ldots \ldots \ldots \ldots \ldots \ldots \ldots \ldots \ldots \ldots \ldots \ldots$. V. Tavian, Z. Y. Li, M. Sutcliffe, J. Gillard (603)

EECP induced instant shear stress variation-experiment and math modeling

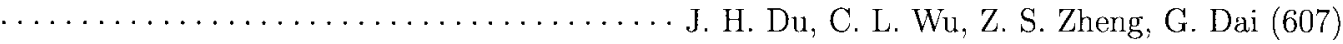

Flow in idealised compliant human cystic duct models

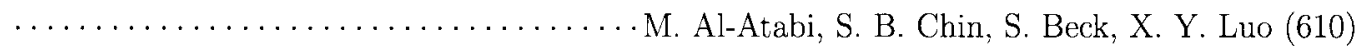

Analysis and comparison of 2-D hemodynamic numerical simulation of elastic aneurysm and rigid aneurysm .... J. W. Zhao, G. H. Ding, W. Y. Yin, X. L. Yang, W. G. Shi, X. L. Zhang (614)

Numerical simulations of blood flow through a permeable curved vessel in a solid tumour

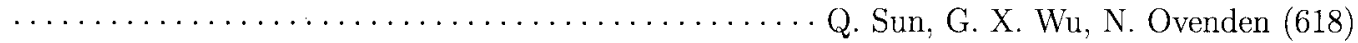

Experimental investigation of blood flow in the brain by means of particle image velocimetry -

a preliminary study $\ldots \ldots \ldots \ldots \ldots \ldots \ldots$ N. A. Buchmann, M. C. Jermy, T. David (622)

Dynamic flight stability of a hovering hoverfly 
Experimental hydrodynamics of turning maneuvers in koi carps

G. Wu, Y. Yang, L. Zeng (630)

Numerical simulations for insect 'clap and fling' with unsteady incompressible solver on dynamic hybrid grids ....... L. P. Zhang, X. H. Chang, X. P. Duan, Z. Y. Wang, H. X. Zhang (634) Preliminary modeling of the fluid-structure interaction on a deformable insect wing in flapping

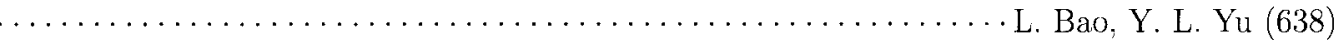

Effects of local configuration on the flow in the circle of willis

J. H. Page, G. X. Wu, F. T. Smith (642)

A numerical investigation of controllably flexible hydrofoil in laminar flows

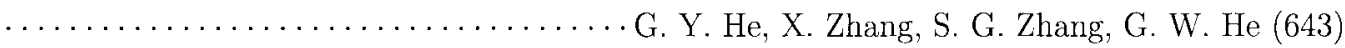

Fish's muscles distortion and pectoral fins propulsion of lift-based mode

S. B. Yang, X. Y. Han, J. Qiu (644)

A numerical study on hydrodynamies of pectoral fin locomotion in Batoid fishes

.W. R. Hu (645)

Effects of temperature on the threshold of phosphorus for algal blooms

T. Yuan, J. C. Li, J. F. Zhou (646)

\section{Micro-scale Flows}

CFD analyses and validation of multiphase flow in micro-fluidic system

.................................... Sewatkar, S. Dindorkar, S. Jadhao (647)

Numerical study on electroosmotic flow in trapezoidal microchannels

C. C. Zuo, F. Ji, L. F. Wang (650)

Lattice Boltzmann simulations for microfluidics and mesoscale phenomena

Mixing flow of viscoelastic fluids in a microchannel

E. Monaco, K. H. Luo, R. S. Qin (654)

......................... Lam, H. Y. Gan, N. T. Nguyen, H. Lie (658)

Direct numerical simulation of micro-flow resonators in the presence of grazing flow

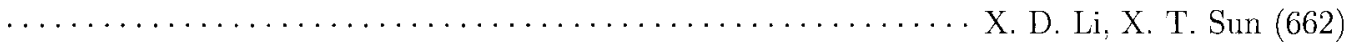

Slip flow regime past over a microsphere

A. Anbarchian, H. Torabian (666)

On the early development of dispersion in flow through a tube with wall reactions

Fully-developed pulsating liquid flows in a three-dimensional wavy wall microtube

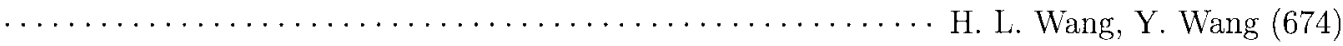

Microreactor system using the concept of numbering-up

$\ldots \ldots \ldots \ldots \ldots \ldots \ldots \ldots \ldots \ldots \ldots \ldots$ S. Togashi, T. Miyamoto, T. Sano, M. Suzuki (678)

Diagnosis of frequency-dependent electrokinetic flow in microfluidic channels

D. G. Yan, C. Yang, X. Y. Huang, Y. C. Lam (682)

Numerical study on flow focusing in axisymmetric microchannel

X. P. Chen $(687)$

Outline of mesoscopic fluid mechanics

G. W. Hu, X. S. Hu (688)

Static threshold pressure gradient of micro-channels 


\section{Plasma and Magneto-Hydrodynamics}

Liquid metal magnetohydrodynamics-_ - astrophysical relevance and engineering applications ......................... Gerbeth, I. Grants, T. Gundrum, F. Stefani (690)

Axisymmetric wave on the charged surface of a viscous liquid

MHD stability of natural convection in rectangular cavity

M. Maache, R. Bessaih (698)

The performance analyse of the pulsed plasma thruster

L. Yin, J. Zhou (702)

Numerical investigation of hypersonic MHD flows

G. Chen, J. B. Zhang, C. H. Lee (706)

Three dimensional interactions between solar wind and magnetopause

Y. Darvish, H. Farman (710)

Characteristics of flow field in an electromagnetic flowmeter for measuring flowrate in injection

polymer well $\cdots \cdots \cdots \cdots \cdots \cdots$. G. B. Zheng, N. 'D. Jin, J. Y. Guo, Y. H. Zhang, J. Li (714)

Control of a magnetic fluid drop moving inside a cylinder

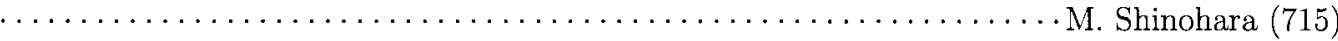

Hydrodynamic analog for electromagnetic structures of sprites

K. H. W. Chu (716)

Stability and numerical simulation of the liquid metal pinch using the shallow water approximation

B. W. Li, E. Zienicke, A. Thess, A. Kräzschmar, P. Terhoeven (717)

\section{Supplement}

Stability analysis for the immersed boundary method

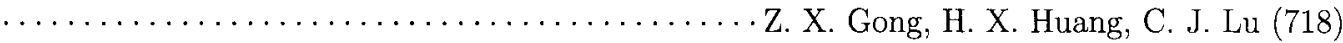

On vorticity decomposition

Symplectic analytical solution for Stokes flow in an annular cavity Y. Wang, Z. C. Deng (726)

A new second order mimetic finite difference scheme to tackle boundary layers-like problems

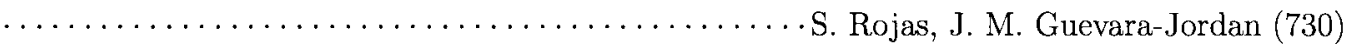

Dual variational principles for 3-D Navier-Stokes equations

Experimental investigation of ventilated supercavitating flows manipulated by a pitching system

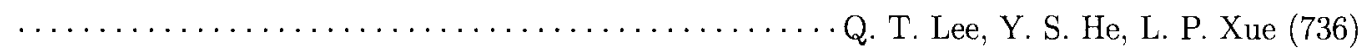

Simulation of the flow field characteristics of a downburst

................ Cao, Y. Zhao, H. Ozono, Y. Tamura, A. Kareem, Z. Duan (740)

\section{Author Index}

